\title{
Antitumor activity of fermented noni exudates and its fractions
}

\author{
JINHUA LI ${ }^{1,5}$, LENG-CHEE CHANG ${ }^{2}$, MARISA WALL ${ }^{3}$, D.K.W. WONG ${ }^{4 *}$, \\ XIANZHONG $\mathrm{YU}^{1}$ and YANZHANG WEI ${ }^{1}$
}

\author{
${ }^{1}$ Department of Biological Sciences, Clemson University, Clemson, SC $29634 ;{ }^{2}$ College of Pharmacy, \\ University of Hawaii-Hilo; ${ }^{3}$ US Department of Agriculture, Agricultural Research Service, United States Pacific \\ Basin Agricultural Research Center Hilo, HI 96720; ${ }^{4}$ Department of Alternative and Complementary Medicine, \\ John Burns School of Medicine, University of Hawaii-Manoa, Honolulu, HI 96822, USA
}

Received June 29, 2012; Accepted September 13, 2012

DOI: $10.3892 / \mathrm{mco} .2012 .24$

\begin{abstract}
Noni has been extensively used in folk medicine by Polynesians for over 2000 year. Recent studies have shown that noni has a wide spectrum of therapeutic activities including inhibition of angiogenesis, anti-inflammatory effects and anticancer activities. Intraperitoneal (i.p.) injection of fermented noni exudates (fNE) were previously found to induce significant tumor rejection in a $\mathrm{S} 180$ mouse sarcoma tumor model, while natural killer (NK) cells were demonstrated to be markedly involved in fNE-induced antitumor activity. In this study, fNE was partitioned into three fractions and their antitumor effects were examined using i.p. injection or as water supplement. The in vivo animal study results showed that when delivered by i.p. injection, n-butanol fraction of fNE $(\mathrm{BuOH})$ effectively rejected $(100 \%)$ tumor challenge and eradicated existing tumors $(75 \%)$. When delivered as a water supplement, $62.5 \%$ of the mice receiving the n-butanol or ethyl acetate fractions resisted tumor cells. The tumor-resistant mice effectively rejected more and higher doses of tumor challenge, indicating that the immune system was activated. The findings confirm those of an earlier study showing fNE to have antitumor activity and demonstrating that the n-butanol fraction of fNE contains active antitumor components, to be further identified. More importantly, the antitumor effect of fNE and its fractions as water supplements renders a significant potential for identifying novel and powerful new dietary products for cancer prevention.
\end{abstract}

Correspondence to: Dr Yanzhang Wei, Department of Biological Sciences, Clemson University, 132 Long Hall, Clemson, SC 29634, USA

E-mail: ywei@clemson.edu

Present address: ${ }^{5}$ Greenville College of Technology, Greenville, SC 29651, USA

\section{"Deceased}

Key words: noni, fermented noni exudates, cancer

\section{Introduction}

Noni (Morinda citrifolia) is a small evergreen tree native to South Asia, with elongated leaves, white tubular flower clusters and greenish fruits that ripen to whitish-yellow. Its products have been used for centuries to treat an array of maladies. A variety of major natural products have been identified in the noni plant thus far $(1,2)$. Two novel glycosides and a new unusual iridoid, known as citrifolinoside, isolated from noni were shown to have an inhibiting effect on activator protein-1 (AP-1) transactivation and cell transformation in the mouse epidermal JB6 cell line (3-7). Although its traditional uses primarily involved topical application of leaves and roots, the noni fruit has been more popular for modern usage (8). The documented effects of noni juice include anti-inflammatory, antiangiogenic, anticancer, antibacterial and antioxidant activities. Noni has been reported to modulate immune cells and has been found to enhance the adaptive immune response by activating $\mathrm{T}$ and $\mathrm{B}$ cells (9).

Noni has received much attention for its antiangiogenic and anticancer properties in animal models and clinical settings. The first attempt to identify the component of noni responsible for its antitumor activities described a water-soluble, ethanol-precipitating, polysaccharide-rich substance from noni fruit, as an immunomodulator (10). Shortly thereafter, Wang and Su demonstrated that noni inhibits 7,12-dimethylbenz[a]anthracene (DMBA)-DNA adduct formation (11), suggesting a protective role in chemically induced cancers. In their study, Furusawa et al expanded their investigation to demonstrate that the antitumor properties of noni precipitate (ppt) were dependent on macrophages, NK and T cells and extended its synergistic effects with even more chemotherapies (12). Additionally, noni was found to inhibit new vessel sprouts at concentrations of 5\% and induce vessel degeneration at a concentration of $10 \%$ (13). In 2004 , Wong developed a novel technique of making fermented noni exudates (fNE) and reported that two cancer patients had benefited by drinking fNE (14).

The intraperitoneal injection (i.p.) of C57BL/6J mice with fNE has recently been reported (15) to significantly increase the percentages of granulocytes and NK cells in the peripheral blood, peritoneum and spleen. The fNE injection induced complete tumor rejection in normal C57BL/6J mice in both 
preventive and therapeutic treatment settings. Furthermore, that study also showed that fNE injection induced partial tumor rejection in C57 nude mice lacking functional lymphocytes, although no tumor rejection was detected in NK cell-deficient beige mice. Over $85 \%$ of the $\mathrm{C} 57 \mathrm{BL} / 6 \mathrm{~J}$ mice that received fNE survived the first tumor injection and rejected up to $5 \times 10^{6}$ tumor cells when re-challenged. These data demonstrate that fNE is likely to stimulate the innate and adaptive immune systems to reject tumor cells. NK cells exhibit a rapid response and are fundamentally involved in the innate immune system, while a response in the adaptive immune system occurs later with a retained memory (15). This conclusion has been supported in a recent study by Nayak and Mengi (16).

Therefore, to identify the active antitumor components from $\mathrm{fNE}$, the juice was partitioned into three fractions and their antitumor activities were examined in a S180 sarcoma tumor model using either i.p. injection or as a water supplement. The in vivo animal study results showed that n-butanol fraction of $\mathrm{fNE}(\mathrm{BuOH})$ is the most effective component in the two experimental settings.

\section{Materials and methods}

Cells and mice. C57BL/6J mice (female) at 6-8 weeks of age were purchased from Jackson Laboratories (Bar Harbor, ME, USA) and housed in our pathogen-free animal facilities. The animal experiments were carried out in accordance with the Guidelines for the Care and Use of Laboratory Animals (NIH Publication No. 85-23) and the institutional guidelines. Mouse sarcoma S180 tumor cells were purchased from ATCC (Manassas, VA, USA) (TIB-66) and cultured in Dulbecco's modified Eagle's medium (DMEM) with $10 \%$ fetal bovine serum (FBS) and gentamicin $(50 \mu \mathrm{g} / \mathrm{ml})$.

Preparation of fNE and its fractions. Fermented noni exudates (fNE) were prepared as described previously (15). Briefly, physiologically mature, firm-yellow (ripe) noni fruits were harvested from a commercial orchard on the island of Hawaii and transported to the USDA-ARS Laboratory (Hilo, HI, USA) in a cooler. Fruit were of excellent quality (disease-and injury-free). Fruit were destemmed, sorted, washed, sanitized and kept at $22^{\circ} \mathrm{C}$ until translucent. The jars, sieves and utensils used for juice preparation were autoclaved for sterility. Fruit (1.5-1.7 kg/jar) were placed into sterilized glass jars (3.8 liters) with sealed lids to provide low oxygen conditions and allowed to ferment for 14 days at $22^{\circ} \mathrm{C}$. For each batch, $20-25$ jars of noni were prepared. Gas concentrations $\left(\mathrm{CO}_{2}\right.$ and $\left.\mathrm{O}_{2}\right)$ inside jars were measured using a headspace analyzer (model 6600, Illinois Instruments, Johnsburg, IL, USA). Jars were fitted with septa for gas sampling. By day 14, the $\mathrm{CO}_{2}$ levels increased to $25 \%$, whereas $\mathrm{O}_{2}$ levels decreased to $10 \%$. The noni exudates were decanted through a sterile mesh screen, collected in sterile flasks, pasteurized at $85^{\circ} \mathrm{C}$ in a water bath for $3 \mathrm{~min}$, cooled and frozen at $-20^{\circ} \mathrm{C}$. The noni juice had a pH of 3.5 and a total soluble solids content of $5.7 \%$.

Fresh or thawed fNE were partitioned with ethyl acetate to obtain the ethyl acetate-soluble residue. Then, the remaining aqueous layer was partitioned three times with n-butanol. Thus, two organic fractions designated as $\mathrm{EtAc}$ and $\mathrm{BuOH}$ and an aqueous fraction $\left(\mathrm{H}_{2} \mathrm{OL}\right)$ were obtained. The three fractions were freeze-dried in a lyophilizer and sent via overnight air shipment to the Clemson University for evaluation in their antitumor assays. Unfractionated fNE was also shipped. Prior to injection, the noni fractions were thawed and dissolved with phosphate-buffered saline (PBS) to one fourth of the original fNE volume and adjusted to $\mathrm{pH}$ 7.0-7.4. The $\mathrm{H}_{2} \mathrm{O}$ fraction was later found to be toxic. Therefore, fNE, $\mathrm{BuOH}$ and EtAc fractions were used in the animal study.

Animal studies. Three animal studies were conducted with the noni materials. In a tumor prevention study, four cages of female C57BL/6J mice ( $\mathrm{n}=8 / \mathrm{cage}$; age, 10 weeks) were i.p. injected with $0.2 \mathrm{ml} / \mathrm{mouse}$ of $\mathrm{fNE}, \mathrm{BuOH}$ and EtAc fractions or PBS on days 14 and 7. On day $0,5 \times 10^{5}$ S180 tumor cells were injected i.p. into each mouse and their survival was monitored. In a tumor treatment study, C57BL/6J mice were injected i.p. with live S180 tumor cells $\left(5 \times 10^{5}\right.$ cells/mouse) on day 0 . On days 3,4 and 5, the mice were i.p. injected with $0.2 \mathrm{ml}$ of fNE, $\mathrm{BuOH}$ and EtAc fractions or PBS ( $\mathrm{n}=8$ mice/group). The survival of the mice was monitored. In a tumor prevention study with noni materials as water supplements, female C57BL/6J mice (age, 8 weeks) were fed with water containing fNE, $\mathrm{BuOH}$ or EtAc fractions at a concentration of $5 \%$ for 4 weeks ( $n=4$ mice/group). The mice were then injected i.p.

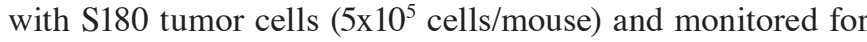
survival.

\section{Results}

fNE fractions. Thawed fNE $(400 \mathrm{ml})$ were partitioned with EtAc three times ( $3 \times 200 \mathrm{ml})$ to obtain the EtAc-soluble residue. Solvent was thoroughly evaporated under reduced pressure using a rotary evaporator. One gram (dry weight) of residue was obtained from this process and labeled as EtAc fraction. The remaining aqueous layer was partitioned with n-butanol three times (3x200 ml). Nineteen grams (dry weight) of residue were obtained and labeled as $\mathrm{BuOH}$ fraction. Twenty grams (dry weight) of residue were obtained from the aqueous layer and labeled as $\mathrm{H}_{2} \mathrm{OHL}$. The dry noni materials were dissolved in $100 \mathrm{ml} \mathrm{PBS} \mathrm{(} 1 / 4$ of the original fNE volume) and adjusted to $\mathrm{pH}$ 7.2-7.4. A pilot study showed that $\mathrm{H}_{2} \mathrm{OHL}$ was toxic (data not shown), therefore, fNE, BuOh and EtAc were used in the subsequent in vivo animal studies.

Intraperitoneal injection of noni materials rejected 5180 tumor challenge. Four cages of female C57BL/6J mice ( $\mathrm{n}=8 /$ cage; age, 10 weeks) were i.p. injected with $0.2 \mathrm{ml} / \mathrm{mouse}$ of fNE, BuOH and EtAc fractions or PBS on days 14 and 7. On day $0,5 \times 10^{5} \mathrm{~S} 180$ tumor cells were injected i.p. into each mouse and their survival was monitored. While the injection of PBS or EtAc did not provide any protection from S180 tumor challenge, the mice injected with $\mathrm{BuOH}$ fraction completely rejected S180 tumor challenge. Six of eight mice injected with fNE rejected S180 tumor cells (Fig. 1). These mice remained tumor-free for their entire lifespan. Two months later, four tumor cell-resistant mice from the fNE and $\mathrm{BuOH}$ groups were rechallenged with $5 \times 10^{6}$ live S180 tumor cells/mouse i.p. The mice completely rejected this second tumor cell challenge, indicating that an effective immune response had been activated. 


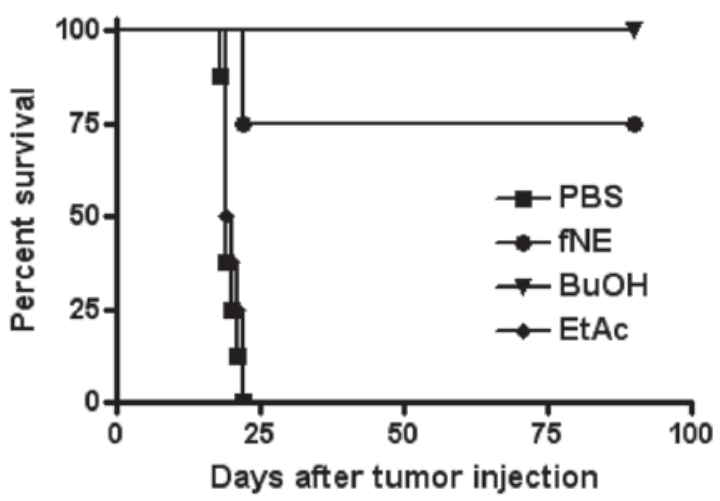

Figure 1. C57BL/6J mice ( $=8$ ) were i.p. injected with $0.2 \mathrm{ml} / \mathrm{mouse}$ of fNE, BuOH or PBS, respectively, on days 14 and 7. On day $0,5 \times 10^{5} \mathrm{~S} 180$ tumor cells were i.p. injected into each mouse and the survival of the mice was monitored. Mice injected with $\mathrm{BuOH}$ fraction completely rejected S180 tumor challenge. Six of eight mice injected with fNE rejected S180 tumor cells.

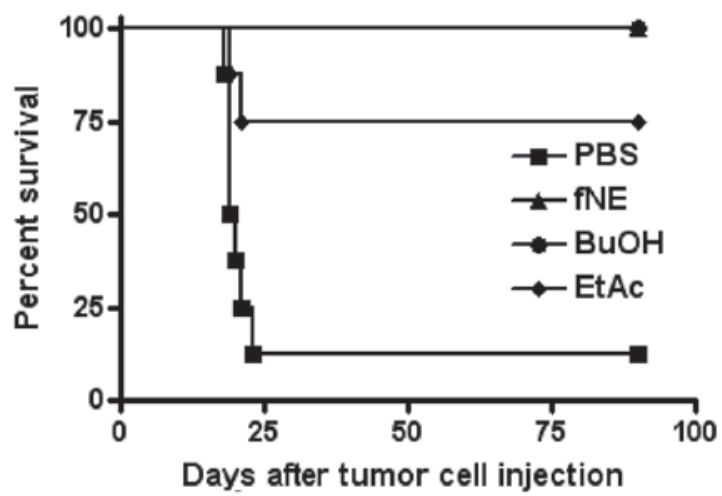

Figure 2. C57BL/6J mice ( $\mathrm{n}=8$ ) were i.p. injected with live $\mathrm{S} 180$ tumor cells ( $5 \times 10^{5}$ cells/mouse) on day 0 . On days 3,4 and 5, the mice were i.p. injected with $0.2 \mathrm{ml}$ of $\mathrm{fNE}, \mathrm{BuOH}, \mathrm{EtAc}$ or PBS, respectively. The survival of the mice was monitored.The i.p. injection of $\mathrm{fNE}$ and $\mathrm{BuOH}$ fraction completely eradicated the existing S180 tumor cells, while six of the eight mice injected with the EtAc fraction were tumor-free

Intraperitoneal injection of noni materials eradicated existing S180 tumor cells. In a therapeutic treatment experimental setting, C57BL/6J mice were injected i.p. with live S180 tumor cells ( $5 \times 10^{5}$ cells/mouse). On days 3, 4 and 5, the mice were i.p. injected with $0.2 \mathrm{ml}$ of $\mathrm{fNE}, \mathrm{BuOH}$ fractions, EtAc fractions or PBS ( 8 mice/group). The survival of the mice was monitored. The i.p. injection of $\mathrm{fNE}$ and $\mathrm{BuOH}$ fraction completely eradicated the existing S180 tumor cells, while six of the eight mice injected with the EtAc fraction were tumor-free (Fig. 2).

Mice fed with noni materials as a water supplement rejected S180 tumor challenge. Female C57BL/6J mice (age, 8 weeks) were fed with water containing $\mathrm{fNE}, \mathrm{BuOH}$ or EtAc fractions at a concentration of $5 \%$ for 4 weeks ( $n=8$ mice/group). The mice were then injected i.p. with S180 tumor cells $\left(5 \times 10^{5}\right.$ cells/mouse) and monitored for survival. Six of eight mice fed with fNE rejected S180 tumor challenge, five of eight mice fed with $\mathrm{BuOH}$ or four of eight mice fed with EtAc rejected S180 tumor challenge. The mice that survived

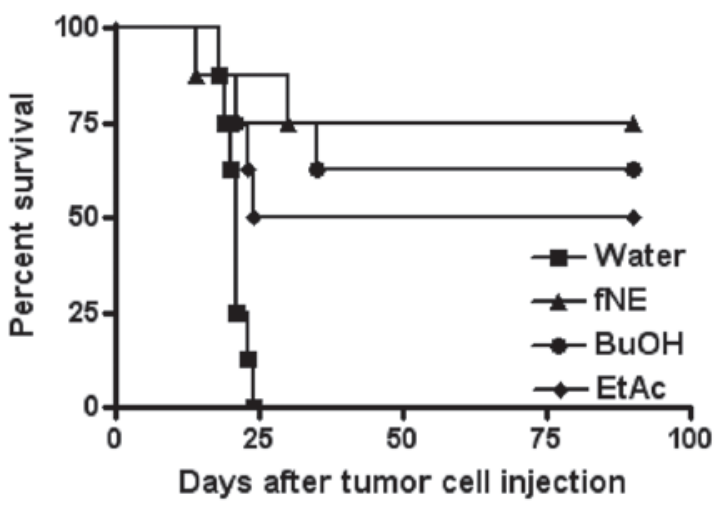

Figure 3. Female C57BL/6J mice $(n=8)$ were fed with water containing fNE, BuOH or EtAc, respectively, at a concentration of $5 \%$ for 4 weeks ( 8 mice/group). The mice were then i.p. injected with S180 tumor cells $\left(5 \times 10^{5}\right.$ cells/mouse) and monitored for survival. Six of eight mice fed with fNE rejected S180 tumor challenge, five of eight mice fed with $\mathrm{BuOH}$ or four of eight mice fed with EtAc rejected S180 tumor challenge. The mice that survived the first tumor challenge were tumor-free for their entire lifespan.

the first tumor challenge were tumor-free for their entire lifespan (Fig. 3).

\section{Discussion}

Although noni products have been used as folk medicines for thousands of years, it is only recently that the mechanisms and the active components of noni have been understood, with attention being focused on noni juice $(10,12)$. The method to produce noni juice was modified by the addition of fermentation and the product was termed fermented noni exudates (fNE) $(14,15)$. Novel species were identified from fNE (17). fNE was demonstrated to be used to prevent tumor development as well as treat existing tumors in a mouse sarcoma tumor model. This antitumor activity is mediated by the activation of the immune system, especially by NK cells (15). The fNE was also shown to have the potential to activate dendritic cells that are important in the initiation of the adaptive immunity (18).

As the first step in identifying the active antitumor components or compounds from fNE in this study, the noni juice was partitioned into three fractions: water $\left(\mathrm{H}_{2} \mathrm{OL}\right)$, alcohol $(\mathrm{BuOH})$ and acid (fractions EtAc). The antitumor activities of these fractions were then examined in an in vivo S180 sarcoma tumor model using either i.p. injection or as a water supplement. $\mathrm{H}_{2} \mathrm{OL}$ was found to be extremely toxic due to the contamination of lipopolysaccharide (LPS) (data not shown) and not included in the in vivo assay.

In a preventive setting, $\mathrm{fNE}, \mathrm{BuOH}$ or EtAc were i.p. injected into experimental mice in order to determine whether or not the noni fractions stimulated the immune system to reject tumor cells. As shown in Fig. 1, BuOH stimulated a stronger immune response compared to fNE, since the eight mice that received $\mathrm{BuOH}$ injection rejected $\mathrm{S} 180$ tumor cells, while six of the mice that received fNE rejected the tumor cells. Of note, no immune response was stimulated subsequent to EtAc injection. Consequently, the active antitumor compounds are very likely present in the alcohol fraction of fNE. More importantly, the mice that received $\mathrm{fNE}$ or $\mathrm{BuOH}$ and rejected the first tumor challenge rejected a second and higher dose of 
tumor challenge, indicating that long-term immunomemory was established (data not shown).

To determine whether or not the antitumor immune response activated by fNE and its fractions has the potential to eradicate existing tumor cells, mice were injected with tumor cells and then treated with $\mathrm{fNE}, \mathrm{BuOH}$ or EtAc. As shown in Fig. 2, the mice treated with $\mathrm{fNE}$ or $\mathrm{BuOH}$ eradicated tumor cells and remained tumor-free for their entire lifespan. Notably, six of the eight mice treated with EtAc also exhibited eradicated tumor cells. At this point of the experiment, two phenomena were of interest. First, the therapeutic treatment showed a better antitumor activity compared to the preventive treatment. Secondly, EtAc treatment did not reject tumor challenge, but was able to eradicate existing tumors. A possible explanation for these noteworthy observations is the activation of the two arms of the immune system: innate and adaptive immunity. Although the immunities might be activated in the preventive setting, only adaptive immunity has the potential to generate immunomemory, which rejects a future tumor challenge. In the therapeutic treatment setting, the immunities are activated and mount a rapid and effective immune response against the tumor cells. In addition, EtAc may not be effective in stimulating the adaptive immune responses, but is able to activate the innate immune response, especially the NK cells. These interesting findings require further investigation.

Since the i.p. injection of fNE and its fractions stimulate the adaptive immunity and reject a later tumor challenge, it was then hypothesized that if the noni materials can be delivered in a natural manner, such as oral delivery, an immunomemory may be established. The fNE, BuOH or EtAc were added into the feeding water for the mice. After a four-week feeding with the noni material-containing water, the mice were i.p. injected with live S180 tumor cells. Fig. 3 shows that the addition of $\mathrm{fNE}, \mathrm{BuOH}$ or EtAc into the feeding water effectively activated the immune system and rejected tumor challenge, although not as effectively as the delivery by direct i.p. injections. Notably, $50 \%$ of the mice fed with EtAc-containing water rejected tumor cells. To determine the reason for which the i.p. injection of the EtAc fraction did not reject tumor challenge as opposed to its oral delivery, additional studies are required.

In conclusion, this preliminary study confirms that fNE is able to activate immune responses to reject tumor challenge as well as eradicate existing tumors. The alcohol fraction $(\mathrm{BuOH})$ of fNE contains the active components that can activate both innate and adaptive immune responses. More importantly, the noni materials may be delivered orally and activate the immune system, representing a novel approach for cancer prevention.

\section{Acknowledgements}

The authors would like to thank Eric Holle and his staff for the professional care of mice used in this study. This study was supported in part by the Patient-First Cancer Foundation (2007639).

\section{References}

1. Balakrishna S, Seshadri TR and Venkataramani B: Special chemical component of commercial woods and related plant materials: Part X-Heartwood of Morinda citrifolia Linn. J Sci Industrial Res 20B: 331-333, 1961.
2. Potterat $\mathrm{O}$ and Hamburger M: Morinda citrifolia (noni) fruit phytochemistry, pharmacology, safety. Planta Med 73: 191-199, 2007.

3. Wang M, Kikuzaki H, Jin Y, Nakatani N, Zhu N, Csiszar K, et al: Novel glycosides from noni (Morinda citrifolia). J Nat Prod 63: 1182-1183, 2000.

4. Sang S, He K, Liu G, Zhu N, Cheng X, Wang M, et al: A new unusual iridoid with inhibition of activator protein-1 (AP-1) from the leaves of Morinda citrifolia L. Org Lett 3: 1307-1309, 2001.

5. Sang S, Cheng X, Zhu N, Wang M, Jhoo JW, Stark RE, et al: Iridoid glycosides from the leaves of Morinda citrifolia. J Nat Prod 64: 799-800, 2001.

6. Sang S, Cheng X, Zhu N, Stark RE, Badmaev V, Ghai G, et al: Flavonolglycosides and novel iridoid glycoside from the leaves of Morinda citrifolia. J Agric Food Chem 49: 4478-4481, 2001.

7. Liu G, Bode A, Ma WY, Sang S, Ho CT and Dong Z: Two novel glycosides from the fruits of Morinda citrifolia (noni) inhibit AP-1 transactivation and cell transformation in the mouse epidermal JB6 cell line. Cancer Res 61: 5749-5756, 2001.

8. Pawlus AD and Kinghorn AD: Review of the ethnobotany, chemistry, biological activity and safety of the botanical dietary supplement Morinda citrifolia (noni). J Pharm Pharmacol 59: 1587-1609, 2007.

9. Schafer M, Sharp P, Brooks VJ, Xu J, Cai J, Keuler NS, Peek SF, Godbee RG, Schultz RD and Darien BJ: Enhanced bactericidal activity against Escherichia coli in calves fed Morinda citrifolia (noni) puree. J Vet Int Med 22: 499-502, 2008.

10. Hirazumi A and Furusawa E: An immunomodulatory polysaccharide-rich substance from the fruit juice of Morinda citrifolia (noni) with antitumour activity. Phytother Res 13: 380-387, 1999.

11. Wang MY and Su C: Cancer preventive effect of Morinda citrifolia (noni). Ann N Y Acad Sci 952: 161-168, 2001.

12. Furusawa E, Hirazumi A, Story S and Jensen J: Antitumour potential of a polysaccharide-rich substance from the fruit juice of Morinda citrifolia (noni) on sarcoma 180 ascites tumour in mice. Phytother Res 17: 1158-1164, 2003.

13. Hornick CA, Myers A, Sadowska-Krowicka H, Anthony CT and Woltering EA: Inhibition of angiogenic initiation and disruption of newly established human vascular networks by juice from Morinda citrifolia (noni). Angiogenesis 6: 143-149, 2003.

14. Wong DK: Are immune responses pivotal to cancer patient's long term survival? Two clinical case-study reports on the effects of Morinda citrifolia (noni). Hawaii Med J 63: 182-184, 2004.

15. Li J, Stickel S, Bouton-Verville H, Burgin KE, Yu X, Wong DK, Wagner TE and Wei Y: Fermented noni exudate (fNE): A mediator between immune system and anti-tumor activity. Oncol Rep 20: 1505-1510, 2008.

16. Nayak $S$ and Mengi $S$ : Immunostimulant activity of noni (Morinda citrifolia) on T and B lymphocytes. Pharmal Biol 48: 724-731, 2010.

17. Nishijima KA, Wall MM, Chang LC, Wei Y and Wong DKW: First report of association of Mucor circinelloides on noni (Morinda citrifolia) in Hawaii. Plant Dis 95: 360, 2011.

18. Zhang X, Li J, Wong DK, Wagner TE and Wei Y: Fermented noni exudate-treated dendritic cells directly stimulate B lymphocyte proliferation and differentiation. Oncol Rep 21: $1147-1152,2009$. 